\title{
ACTUATOR FAILURE COMPENSATION FOR TWO LINKED 2WD MOBILE ROBOTS BASED ON MULTIPLE-MODEL CONTROL
}

\author{
YAJIE MA $^{a}$, VINCENT COCQUEMPOT $^{b, *}$, MAAN EL BADAOUI EL NAJJAR $^{b}$, BIN JIANG ${ }^{a}$ \\ ${ }^{a}$ College of Automation Engineering \\ Nanjing University of Aeronautics and Astronautics, Nanjing 210016, China \\ ${ }^{b}$ UMR 9189-CRIStAL: Research Center in Computer Science, Signal and Automatic Control of Lille \\ University of Lille, CNRS, Centrale Lille, Lille F-59000, France \\ e-mail: vincent.cocquempot@univ-lille1.fr
}

\begin{abstract}
This paper develops a new actuator failure compensation scheme for two linked two-wheel drive (2WD) mobile robots based on multiple-model control. First, a configuration of two linked 2WD robots is described, and their kinematics and dynamics are modeled. Then, a multiple-model based failure compensation scheme is developed to compensate for actuator failures, consisting of a kinematic controller, multiple dynamic controllers and a control switching mechanism, which ensures system stability and asymptotic tracking properties. Finally, simulation results verify the effectiveness of the proposed failure compensation control system.
\end{abstract}

Keywords: actuator failure, multiple-model control, physically linked robots, two-wheel drive.

\section{Introduction}

The demand for wheeled mobile robots is increased in many places, such as shopping centers, hospitals, warehouses, agriculture and nuclear waste facilities (Dixon et al., 2001). In some harsh environments, such as fires, nuclear accidents, natural or industrial disasters, and terrorist attacks, wheeled mobile robots are employed to help rescuers. These robots have various missions: they may carry some automated manipulation instruments like mechanical arms equipped with grippers, environment sensors like cameras or sonar, and also materials for rescuers like tools or medicine. However, the adverse environments increase the probability of faults, including actuator faults, sensor faults and component faults, which may lead to losing the robots and the important instruments and materials they carry. If we send a team of mobile robots, each of them bears the same risk of failing, and if one robot is faulty, we will lose it. A failed robot becomes an obstacle for the others and for the rescuers, which makes the rescue mission more difficult and less efficient.

To handle these faults and make the failed robot

\footnotetext{
${ }^{*}$ Corresponding author
}

continue working (or simply to evacuate it), robots may be physically linked. Mobile robots may be linked initially, or they may be physically independent and linked only when needed. Physical links between robots are essential for solving many collective tasks. For example, robots can form pulling chains to bring a heavy object. Also, during navigation on a rough terrain, physical links can help robots to pass over a large hole or through a steep. More importantly, linked robots provide actuator and sensor redundancies, which improve fault tolerance of the system. If some robots are faulty, others may be used to link them and help them continue moving. Using this concept of linked robots makes the multi-robot system more flexible, more efficient and more robust with respect to faults. This paper is concerned with fault tolerant control of such linked wheeled multi-robot systems with actuator failures, which is performed in the context of the project SUCRé from the Hauts-de-France region in France, whose objective is to use robots in collaboration with humans (rescuers) in crisis situations.

There are a lot of research results on fault diagnosis and fault tolerant control for various applications (e.g., Ye and Yang, 2006; Blanke et al., 2006; Zhang and Jiang, 2008; Zou and Kumar, 2012; Li and Yang, 2012; 
Efimov et al., 2013; Patton et al., 2012; Yu and Jiang, 2015; Franzè et al., 2015; Hamayun et al., 2015; Yang and Maciejowski, 2015; Rotondo et al., 2015; Bilski and Wojciechowski, 2016; Hassanabadi et al., 2016). As for the application to wheeled mobile robots, some fault diagnosis methods are developed (e.g., Fourlas et al., 2015; Goel et al., 2000; Skoundrianos and Tzafestas, 2004), a sensor fault accommodation scheme is presented by Ji and Sarkar (2007), some fault-tolerant control systems are designed by Koh et al. (2012), Zhang and Cocquempot (2014), Rotondo et al. (2014), Kim et al. (2015), and Aref et al. (2015) for four-wheel drive robots, and a hybrid fault adaptive control scheme is designed by Ji et al. (2003) to accommodate partial faults and degradation for two-wheel drive (2WD) mobile robots. However, even if it is important to compensate a partial loss of wheel-motor effectiveness, the actuators (motors) of a wheeled mobile robot may also be totally faulty.

There are several types of wheeled robots (e.g., Campion et al., 1996; Caracciolo et al., 1999; Fukao et al., 2000; Kozłowski and Pazderski, 2004; Canudas de Wit et al., 2012; González-Sierra et al., 2014; Yang et al., 2016). One often used is the 2WD mobile robot (e.g., Fierro and Lewis, 1995; Ge et al., 2003; Do et al., 2004; Huang et al., 2014). A 2WD robot has two motors with no redundant actuator. When one motor is lost, the $2 \mathrm{WD}$ robot becomes uncontrollable. Thus it is important to improve fault tolerance (using physical links) for 2WD robots. On the other hand, even for a four-wheel drive (4WD) mobile robot that has redundant actuators, some actuator failures may also result in making the robot uncontrollable. Several 4WD robots can also be linked to deal with such faults.

For robot systems with multiple linked wheeled bodies, a feedback nonlinear control scheme is given by Canudas de Wit et al. (1997) for a train-like vehicle; several kinematic control methods are proposed by Sørdalen and Wichlund (1993), Tilbury et al. (1995), Morin and Samson (2012), Michałek (2014; 2017), Akhtar et al. (2015) and Ritzen et al. (2016) for one tractor with multiple trailers systems; a kinematic control and a dynamic control are given by Khalaji and Moosavian (2014) for a tractor with one trailer, in which, the latter is unactuated; two kinematic control schemes are designed by Yuan et al. (2015) for a double-steering two linked 2WD mobile robots system. However, these control methods are proposed without considering actuator faults.

To our knowledge, there is no research result on fault tolerant control for linked wheeled multi-robot systems. Such a new control problem motivates the present work. In this paper, we design a fault tolerant control scheme for two linked 2WD mobile robots as shown in Fig. 1, with a total loss of actuators (motors). For this configuration, if one or two of the four actuated wheels are faulty, the remaining actuated wheels can continue to move the two robots. The actuator failures considered are uncertain in nature, that is to say, the failed actuator(s) and failed time instance(s) are unknown. These uncertainties significantly change the system structure (control gain) from the inputs to the outputs if a new actuator fails. This paper is focused on developing an actuator failure compensation scheme for two linked 2WD mobile robots, and as the failure situation is uncertain, a multiple-model based control design (e.g., Narendra and Balakrishnan, 1997) is used. The main contributions are as follows:

1. The dynamic model of two linked 2WD mobile robots is proposed, which, together with the kinematic model proposed by Khalaji and Moosavian (2014), is employed to deal with the fault tolerant control problem for 2WD mobile robots.

2. A multiple-model based compensation control scheme is developed to compensate for actuator failures for two linked $2 \mathrm{WD}$ mobile robots as shown in Fig. 1, which can ensure the desired system stability and asymptotic tracking properties despite the presence of actuator failures, including simultaneous multi-failures.

The rest of this paper is as follows. In Section 2, the kinematic and dynamic models of two linked 2WD mobile robots are given, and the actuator failure compensation problem is formulated. In Section 3, a multiple-model failure compensation control scheme is developed. In Section 4 a simulation study is presented to verify its effectiveness. Conclusions are given in Section 5 .

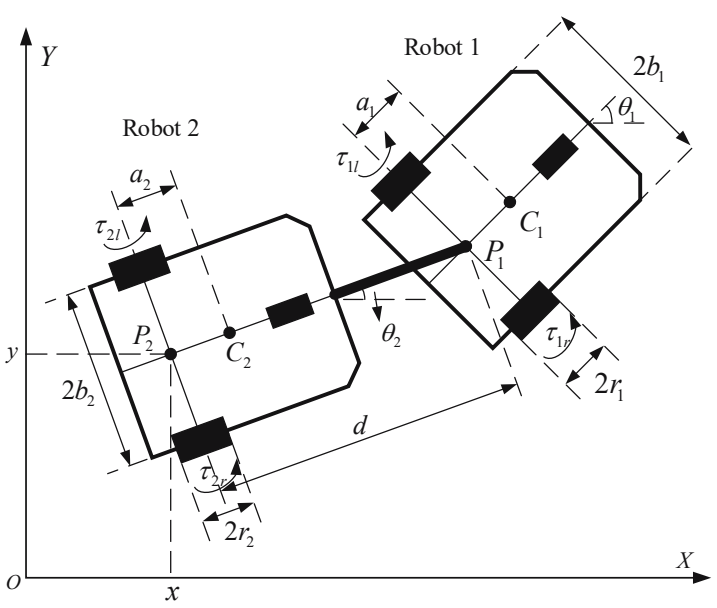

Fig. 1. Two differential 2WD robots with a physical link. 


\section{System modeling and problem formulation}

In this section, the actuator failure compensation problem is formulated for two linked 2WD mobile robots. In Fig. 11 for each robot, the front wheel is passive and the two rear wheels are actuated. For the link, it is fixed at point $P_{2}$ with Robot 2 and connected through a passive rotary joint at $P_{1}$ with Robot 1 . Thus the orientation of Robot 2 is consistent with the link, but the one of Robot 1 is independent.

In this paper, we suppose that (i) the positions, orientations and velocities of the robots are measured by onboard sensors; (ii) the physical parameters of the dynamics are accurately known; (iii) the wheels are driven by actuators through gears of small or unit gear-ratio, which permits wheels rolling despite the failure of motors; (iv) each wheel is a thin solid disk having a single point contact with motion surface; (v) the motion is planar and the two robots have no slipping in the lateral direction.

2.1. Kinematic model. For the $i$-th $(i=1,2)$ robot in Fig. 11 $P_{i}$ is the center between two actuated wheels, $C_{i}$ is the center of mass, $a_{i}$ is the distance between $P_{i}$ and $C_{i}, b_{i}$ is half of the distance between two actuated wheels, $r_{i}$ is the radius of wheels, $\theta_{i}$ is the orientation, and $\tau_{i l}$ and $\tau_{i r}$ are the control torques applied to the left and right actuated wheels, respectively. In addition, $d$ is the distance between $P_{1}$ and $P_{2}, O X Y$ is the inertial frame, $(x, y)$ denotes the position of $P_{2}$ for Robot 2 in frame $O X Y$.

Let $v_{1}$ and $v_{2}$ be the linear velocities of $P_{1}$ and $P_{2}$, which also denote the linear velocities of Robots 1 and 2, respectively. Then we have

$$
v_{2}=v_{1} \cos \left(\theta_{1}-\theta_{2}\right)
$$

It follows that

$$
\dot{\theta}_{2}=\frac{v_{1} \sin \left(\theta_{1}-\theta_{2}\right)}{d}=\frac{v_{2}}{d} \tan \left(\theta_{1}-\theta_{2}\right) .
$$

The kinematic equations of the two linked 2WD mobile robots shown in Fig. 1 are given by

$$
\begin{aligned}
\dot{x} & =v_{2} \cos \theta_{2}, \\
\dot{y} & =v_{2} \sin \theta_{2}, \\
\dot{\theta}_{2} & =\frac{v_{2}}{d} \tan \left(\theta_{1}-\theta_{2}\right), \\
\dot{\theta}_{1} & =\omega_{1},
\end{aligned}
$$

where $\omega_{1}$ is the angular velocity of robot 1 , and $\mid \theta_{1}(0)-$ $\theta_{2}(0) \mid<\pi / 2$.

Let $q:=\left[x, y, \theta_{2}, \theta_{1}\right]^{\top}$ and $\eta:=\left[v_{2}, \omega_{1}\right]^{\top}$. The kinematic equations in (3)- (6) can be rewritten as

$$
\dot{q}=S(q) \eta
$$

where

$$
S(q)=\left[\begin{array}{cccc}
\cos \theta_{2} & \sin \theta_{2} & \frac{1}{d} \tan \left(\theta_{1}-\theta_{2}\right) & 0 \\
0 & 0 & 0 & 1
\end{array}\right]^{\top} .
$$

2.2. System constraints. If we denote by $\left(x_{1}, y_{1}\right)$ the position of $P_{1}$, we have $\dot{x}_{1}=v_{1} \cos \theta_{1}$ and $\dot{y}_{1}=v_{1} \sin \theta_{1}$. Then, according to (3) and (4), the system constraints are

$$
\begin{aligned}
\dot{x}_{1} \sin \theta_{1}-\dot{y}_{1} \cos \theta_{1} & =0, \\
\dot{x} \sin \theta_{2}-\dot{y} \cos \theta_{2} & =0 .
\end{aligned}
$$

With $x_{1}=x+d \cos \theta_{2}$ and $y_{1}=y+d \sin \theta_{2}$, Eqn. (9) can be rewritten as

$$
\dot{x} \sin \theta_{1}-\dot{y} \cos \theta_{1}-\dot{\theta}_{2} d \cos \left(\theta_{1}-\theta_{2}\right)=0 .
$$

Then (9) and (10) can be expressed as

$$
A(q) \dot{q}=0,
$$

where $A(q)$ is the system constraint matrix given by

$$
A(q)=\left[\begin{array}{cccc}
\sin \theta_{1} & -\cos \theta_{1} & -d \cos \left(\theta_{1}-\theta_{2}\right) & 0 \\
\sin \theta_{2} & -\cos \theta_{2} & 0 & 0
\end{array}\right] .
$$

Moreover, with (8) and (13), we have

$$
S^{\top}(q) A^{\top}(q)=0 \text {. }
$$

2.3. Dynamic model. The dynamic model of two linked 2WD robots is obtained by using the Lagrange method without considering the dissipative forces. It gives

$$
M(q) \ddot{q}+E(q, \dot{q})=B(q) \tau+A^{\top}(q) \lambda,
$$

where $M(q) \in \mathbb{R}^{4 \times 4}$ is the inertia matrix that is symmetric positive definite, $E(q, \dot{q}) \in \mathbb{R}^{4}$ is the vector of centrifugal and Coriolis forces, $B(q) \in \mathbb{R}^{4 \times 4}$ is the input injection matrix, $\tau=\left[\tau_{1 r}, \tau_{1 l}, \tau_{2 r}, \tau_{2 l}\right]^{\top}$ is the control torque vector, and $\lambda \in \mathbb{R}^{2}$ is the vector of constraint forces. The matrices and vectors in (15) are given by

$$
\begin{aligned}
& E(q, \dot{q}) \\
& =\left[\begin{array}{c}
-a_{1} m_{1} \dot{\theta}_{1}^{2} \cos \theta_{1}-\left(a_{2} m_{2}+d m_{1}\right) \dot{\theta}_{2}^{2} \cos \theta_{2} \\
-a_{1} m_{1} \dot{\theta}_{1}^{2} \sin \theta_{1}-\left(a_{2} m_{2}+d m_{1}\right) \dot{\theta}_{2}^{2} \sin \theta_{2} \\
-a_{1} d m_{1} \dot{\theta}_{1}^{2} \sin \left(\theta_{1}-\theta_{2}\right) \\
a_{1} d m_{1} \dot{\theta}_{2}^{2} \sin \left(\theta_{1}-\theta_{2}\right)
\end{array}\right], \\
& M(q) \\
& =\left[\begin{array}{cc}
m_{1}+m_{2} & 0 \\
0 & m_{1}+m_{2} \\
-\left(a_{2} m_{2}+d m_{1}\right) \sin \theta_{2} & \left(a_{2} m_{2}+d m_{1}\right) \cos \theta_{2} \\
-a_{1} m_{1} \sin \theta_{1} & a_{1} m_{1} \cos \theta_{1} \\
-\left(a_{2} m_{2}+d m_{1}\right) \sin \theta_{2} & -a_{1} m_{1} \sin \theta_{1} \\
\left(a_{2} m_{2}+d m_{1}\right) \cos \theta_{2} & a_{1} m_{1} \cos \theta_{1} \\
m_{2} a_{2}^{2}+m_{1} d^{2}+I_{m 2} & a_{1} d m_{1} \cos \left(\theta_{1}-\theta_{2}\right) \\
a_{1} d m_{1} \cos \left(\theta_{1}-\theta_{2}\right) & m_{1} a_{1}^{2}+I_{m 1}
\end{array}\right],
\end{aligned}
$$




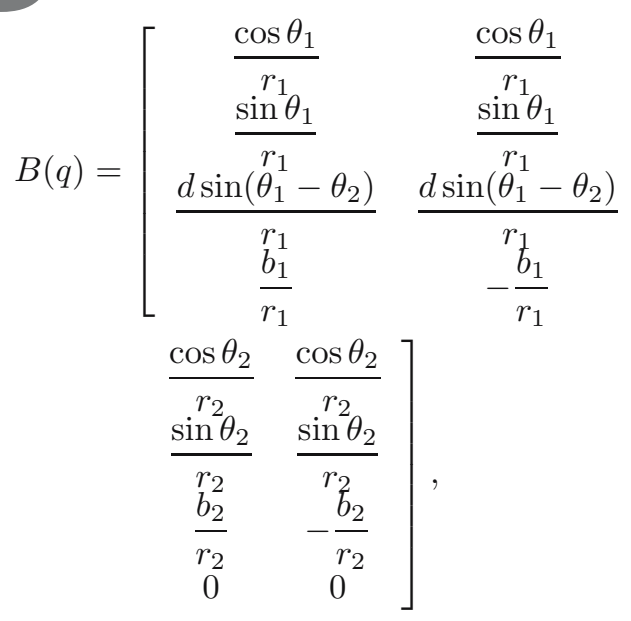

where $m_{1}$ and $m_{2}$ are the masses of the two robots, and $I_{m 1}$ and $I_{m 2}$ denote the inertia parameters with respect to the robots rotating at their centers of mass $\left(C_{1}, C_{2}\right)$ in the planar plane.

Substituting the time derivative of (7) into (15), and multiplying by $S^{\top}(q), A^{\top}(q) \lambda$ can be eliminated with (14). Then (15) becomes

$$
\bar{M}_{1}(q) \dot{\eta}+\bar{M}_{2}(q) \eta+\bar{E}(q, \dot{q})=\bar{B}(q) \tau,
$$

where $\eta=\left[v_{2}, \omega_{1}\right]^{\top}$, and

$$
\begin{aligned}
\bar{M}_{1}(q) & =S^{\top}(q) M(q) S(q), \\
\bar{M}_{2}(q) & =S^{\top}(q) M(q) \dot{S}(q), \\
\bar{E}(q, \dot{q}) & =S^{\top}(q) E(q, \dot{q}), \\
\bar{B}(q) & =S^{\top}(q) B(q) .
\end{aligned}
$$

Note that $\bar{M}_{1}(q)$ is also symmetric positive definite as $M(q)$ is symmetric positive definite.

2.4. Actuator failure model. The actuator failures considered cover the case when some motors lose their powers and cannot generate control torques, which are modeled as

$$
\tau(t)=\sigma(t) u(t),
$$

where $\tau=\left[\tau_{1 r}, \tau_{1 l}, \tau_{2 r}, \tau_{2 l}\right]^{\top}$ is the control torque vector, $u=\left[u_{1 r}, u_{1 l}, u_{2 r}, u_{2 l}\right]^{\top}$ is the control signal vector to be designed, and $\sigma=\operatorname{diag}\left\{\sigma_{1 r}, \sigma_{1 l}, \sigma_{2 r}, \sigma_{2 l}\right\}$ is the uncertain failure pattern matrix with

$$
\sigma_{j}(t)= \begin{cases}0, & \text { if the } j \text {-th motor fails, } \\ 1, & \text { otherwise, }\end{cases}
$$

for $j \in\{1 r, 1 l, 2 r, 2 l\}$. For example, if the motor of the right side in Robot 1 is not faulty, then $\sigma_{1 r}=1$; and if it loses its power, then $\sigma_{1 r}=0$.

2.5. Actuation redundancy. For the two linked $2 \mathrm{WD}$ mobile robots, to control $v_{2}$ and $\omega_{1}$ in (17), there are at least two actuated wheels and the following actuation redundancy condition needs to be satisfied:

$$
\operatorname{rank}(\bar{B} \sigma)=2
$$

for all possible failure pattern matrices $\sigma$.

Remark 1. Recalling $\bar{B}(q)$ in (18), the compensable failure cases satisfying this redundancy condition are as follows: (i) a fault free case, i.e., $\sigma=\operatorname{diag}\{1,1,1,1\}$; (ii) one actuator fails, i.e., $\sigma=\operatorname{diag}\{0,1,1,1\}$, $\sigma=\operatorname{diag}\{1,0,1,1\}, \sigma=\operatorname{diag}\{1,1,0,1\}$, or $\sigma=$ $\operatorname{diag}\{1,1,1,0\}$; (iii) two actuators fail. However, if the two failed actuators are both on Robot 2, i.e., $\sigma=\operatorname{diag}\{1,1,0,0\}$, then the system is similar to a tractor-trailer one (Khalaji and Moosavian, 2014) and is compensable; if two failed actuators are on different robots, i.e., $\sigma=\operatorname{diag}\{0,1,0,1\}, \sigma=\operatorname{diag}\{0,1,1,0\}$, $\sigma=\operatorname{diag}\{1,0,0,1\}$, or $\sigma=\operatorname{diag}\{1,0,1,0\}$, then the system is also compensable; but if the two failed actuators are on Robot 1, the system is noncompensable, because $\operatorname{rank}(\bar{B} \sigma)=1$ with $\sigma=\operatorname{diag}\{0,0,1,1\}$, and there is no torque to control $\omega_{1}$.

2.6. Fault-tolerant control objective. The objective of this paper is to develop an actuator failure compensation scheme for two linked 2WD mobile robots whose model is given by (7) and (17) to asymptotically track a reference trajectory, despite the presence of some actuator failures modeled as (19) and (20) that satisfy the condition (21). In other words, the control objective is to design a control signal $u(t)$ to guarantee that all closed-loop system signals are bounded and $\lim _{t \rightarrow \infty}\left(x(t)-x_{d}(t)\right)=0, \lim _{t \rightarrow \infty}\left(y(t)-y_{d}(t)\right)=0$ and $\lim _{t \rightarrow \infty}\left(\theta_{2}(t)-\theta_{d}(t)\right)=0$ in the presence of actuator failures with an unknown $\sigma(t)$, where $x_{d}, y_{d}, \theta_{d}$ are desired reference trajectories.

Remark 2. The position of $P_{1}$ is determined by $x, y$ and $\theta_{2}$; in this sense, the preceding control objective contains the position objective of the whole system. Moreover, from the dynamic equation in (17), we can see that this objective is implemented by the control of $\omega_{1}$. Together with $\dot{\theta}_{1}=\omega_{1}$, the orientation angle $\theta_{1}$ can be seen as an intermediate variable that needs to be controlled.

In this paper, the desired trajectories are assumed to be implemented by a virtual robot as follows:

$$
\begin{aligned}
& \dot{x}_{d}=v_{d} \cos \theta_{d}, \\
& \dot{y}_{d}=v_{d} \sin \theta_{d}, \\
& \dot{\theta}_{d}=\omega_{d},
\end{aligned}
$$

where $v_{d}$ and $\omega_{d}$ are the linear velocity and angular velocity of the reference virtual robot, respectively. Note that the reference trajectory of the unicycle-like robot can 
be computed based on the differential flatness of these kinematics or by numerical integration of the unicycle kinematic equations. On the one hand, $v_{d}, \omega_{d}, \dot{v}_{d}$ and their time derivatives $\dot{\omega}_{d}, \ddot{v}_{d}$ and $\ddot{\omega}_{d}$ can be computed upon successive time derivatives of $x_{d}, y_{d}$ and $\theta_{d}$; in turn, the desired reference trajectories $x_{d}, y_{d}$ and $\theta_{d}$ can also be computed by integrating (22)- (24) while choosing appropriate $v_{d}, \omega_{d}$ and initial values $x_{d}(0), y_{d}(0), \theta_{d}(0)$, and then $\dot{v}_{d}, \dot{\omega}_{d}, \ddot{v}_{d}$ and $\ddot{\omega}_{d}$ are calculated directly from $v_{d}$ and $\omega_{d}$. In this paper, we consider the tracking problem of mobile robots as described by Fierro and Lewis (1995). Then the following assumption is given for the reference trajectories.

Assumption 1. The reference trajectories $x_{d}, y_{d}$ and $\theta_{d}$ and their third-order time derivatives, as well as the corresponding $v_{d}$ and $\omega_{d}$ and their second-order time derivatives, are continuous and uniformly bounded; moreover, $v_{d} \neq 0$ and $\left|\theta_{2}(0)-\theta_{d}(0)\right|<\pi / 2$.

Remark 3. In Assumption 1 , it is imposed that $v_{d} \neq 0$ to avoid singularity in the controller for trajectory tracking of the mobile robots. On the other hand, in order to stop the vehicle when the tracking mission is completed, another controller could be switched to.

2.7. Design issues. To achieve the control objective, we will develop a multiple-model control scheme covering all possible $\sigma$, which has a cascaded structure as shown in Fig. 2

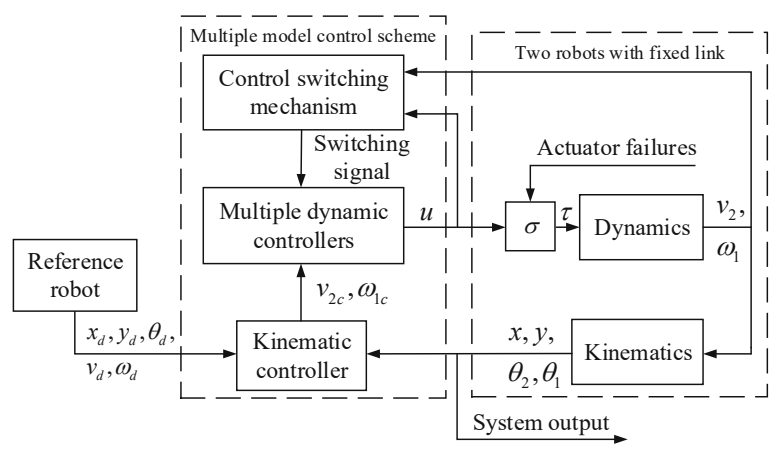

Fig. 2. Block diagram of the multiple-model actuator failure compensation control scheme.

The control design consists of three parts:

(i) For the kinematic equations in (7), $v_{2}$ and $\omega_{1}$ can be seen as intermediate control signals. Thus, we first design a kinematic control law $\eta_{c}=\left[v_{2 c}, \omega_{1 c}\right]^{\top}$ such that, when it is applied, all closed-loop system signals are bounded, and $\lim _{t \rightarrow \infty}\left(x(t)-x_{d}(t)\right)=0, \lim _{t \rightarrow \infty}\left(y(t)-y_{d}(t)\right)=$ 0 and $\lim _{t \rightarrow \infty}\left(\theta_{2}(t)-\theta_{d}(t)\right)=0$.

(ii) Then multiple controllers are designed, each using one possible failure pattern matrix. If the failure pattern used in the controller applied is consistent with the actual one, the employed control signal can ensure $(\eta(t)-$ $\left.\eta_{c}(t)\right) \rightarrow 0$ as $t$ goes to infinity and also the desired system performance.

(iii) Finally, a control switching mechanism is established to select the appropriate controller to generate the employed control signal $u$.

\section{Multiple-model actuator failure compensation scheme}

In this section, a multiple-model failure compensation scheme as shown in Fig. 2 is developed.

\subsection{Kinematic controller design.}

3.1.1. Kinematic control law. Define the output tracking error as

$$
\tilde{e}=\left[\begin{array}{c}
\tilde{e}_{x} \\
\tilde{e}_{y} \\
\tilde{e}_{\theta}
\end{array}\right]:=\left[\begin{array}{c}
x-x_{d} \\
y-y_{d} \\
\theta_{2}-\theta_{d}
\end{array}\right],
$$

and a transformation matrix as

$$
T_{e}\left(\theta_{d}\right):=\left[\begin{array}{ccc}
\cos \theta_{d} & \sin \theta_{d} & 0 \\
-\sin \theta_{d} & \cos \theta_{d} & 0 \\
0 & 0 & 1
\end{array}\right] .
$$

Then, a new error is defined as

$$
e=\left[e_{x}, e_{y}, e_{\theta}\right]^{\top}:=T_{e} \tilde{e} .
$$

Note that, since $T_{e}$ is nonsingular with $\operatorname{det}\left[T_{e}\right]=1$, and if $\lim _{t \rightarrow \infty} e(t)=0$, then $\lim _{t \rightarrow \infty} \tilde{e}(t)=0$. According to (3)-(5), (22)-(24) and (25)-27), we have the time derivatives of $e_{x}, e_{y}, e_{\theta}$ as follows:

$$
\begin{aligned}
& \dot{e}_{x}=\omega_{d} e_{y}+v_{2} \cos e_{\theta}-v_{d}, \\
& \dot{e}_{y}=-\omega_{d} e_{x}+v_{2} \sin e_{\theta}, \\
& \dot{e}_{\theta}=\frac{v_{2}}{d} \tan \left(\theta_{1}-\theta_{2}\right)-\omega_{d} .
\end{aligned}
$$

The details needed to obtain (28)-(30) are given in Appendix A

To develop a kinematic control law $\eta_{c}=\left[v_{2 c}, \omega_{1 c}\right]^{\top}$ for $\eta=\left[v_{2}, \omega_{1}\right]^{\top}$, we introduce the following diffeomorphism:

$$
\begin{aligned}
& z_{1}:=e_{x}, \\
& z_{2}:=e_{y}, \\
& z_{3}:=\tan e_{\theta},
\end{aligned}
$$

with an additional signal

$$
z_{4}:=\frac{\tan \left(\theta_{1}-\theta_{2}\right)}{d \cos ^{3} e_{\theta}}-\frac{\omega_{d}}{v_{d} \cos ^{2} e_{\theta}}+e_{y},
$$


and an input transformation

$$
\alpha=\left[\begin{array}{l}
\alpha_{1} \\
\alpha_{2}
\end{array}\right]:=\left[\begin{array}{c}
v_{2} \cos e_{\theta}-v_{d} \\
\dot{z}_{4}
\end{array}\right] .
$$

Then, the time derivatives of $z_{1}, z_{2}, z_{3}$ and $z_{4}$ are

$$
\begin{aligned}
& \dot{z}_{1}=\omega_{d} z_{2}+\alpha_{1}, \\
& \dot{z}_{2}=-\omega_{d} z_{1}+\left(v_{d}+\alpha_{1}\right) z_{3}, \\
& \dot{z}_{3}=v_{d}\left(z_{4}-z_{2}\right)+\alpha_{1}\left(z_{4}-z_{2}+\frac{\omega_{d}}{v_{d}}\left(1+z_{3}^{2}\right)\right), \\
& \dot{z}_{4}=\alpha_{2} .
\end{aligned}
$$

The details needed to obtain (36) -39 are given in Appendix B

Differentiating (34) and together with (35), we obtain

$$
\alpha=T_{\alpha} \eta+f_{\alpha},
$$

where $T_{\alpha} \in \mathbb{R}^{2 \times 2}$ and $f_{\alpha} \in \mathbb{R}^{2}$ are given in Appendix $\mathrm{C}$. The nonsingularity and boundedness of $T_{\alpha}$ will be analyzed in the performance analysis later.

Define a virtual kinematic control law

$$
\alpha_{c}:=T_{\alpha} \eta_{c}+f_{\alpha}
$$

and the velocity tracking error as

$$
\eta_{e}:=\eta-\eta_{c}
$$

Then we have

$$
\alpha_{e}=\alpha-\alpha_{c}=T_{\alpha} \eta_{e} .
$$

Now, we design the virtual kinematic control law $\alpha_{c}$ as

$$
\begin{aligned}
& \alpha_{c 1}=-k_{1}\left(z_{1}+z_{3}\left(z_{4}+\frac{\omega_{d}}{v_{d}}\left(1+z_{3}^{2}\right)\right),\right. \\
& \alpha_{c 2}=-k_{2} v_{d} z_{3}-k_{3} z_{4},
\end{aligned}
$$

where $k_{1}>0, k_{2}>0$ and $k_{3}>0$ are chosen to be constant. Note that, if $v_{d}$ is too small, then $\alpha_{c 1}$ may be very large, which will result in a bad system transient response. Accordingly, for the practical situation, the reference velocity $v_{d}$ should be chosen as an appropriate one that can contribute to a smooth system transient response. Upon (41), the kinematic control law is

$$
\eta_{c}=T_{\alpha}^{-1}\left(\alpha_{c}-f_{\alpha}\right)
$$

3.1.2. Preliminary analysis. Select a positive definite function as

$$
V_{1}=\frac{1}{2}\left(z_{1}^{2}+z_{2}^{2}+z_{3}^{2}+\frac{1}{k_{2}} z_{4}^{2}\right) .
$$

According to (36)-(39), its time derivative is

$$
\begin{aligned}
\dot{V}_{1}= & z_{1} \omega_{d} z_{2}+z_{1} \alpha_{1}-z_{2} \omega_{d} z_{1}+z_{2} v_{d} z_{3}+z_{2} \alpha_{1} z_{3} \\
& +z_{3} v_{d} z_{4}-z_{3} v_{d} z_{2}+z_{3} \alpha_{1} z_{4}-z_{3} \alpha_{1} z_{2} \\
& +\frac{z_{3} \alpha_{1} \omega_{d}\left(1+z_{3}^{2}\right)}{v_{d}}+\frac{z_{4}}{k_{2}} \alpha_{2} \\
= & \left(z_{1}+z_{3}\left(z_{4}+\frac{\omega_{d}}{v_{d}}\left(1+z_{3}^{2}\right)\right)\right) \alpha_{1}+v_{d} z_{3} z_{4}+\frac{z_{4}}{k_{2}} \alpha_{2} \\
= & \left(z_{1}+z_{3}\left(z_{4}+\frac{\omega_{d}}{v_{d}}\left(1+z_{3}^{2}\right)\right)\right) \alpha_{c 1}+v_{d} z_{3} z_{4} \\
& +\frac{z_{4}}{k_{2}} \alpha_{c 2}+\left(z_{1}+z_{3}\left(z_{4}+\frac{\omega_{d}}{v_{d}}\left(1+z_{3}^{2}\right)\right)\right) \alpha_{e 1} \\
& +\frac{z_{4}}{k_{2}} \alpha_{e 2} .
\end{aligned}
$$

Letting $f_{\eta}:=\left[z_{1}+z_{3}\left(z_{4}+\frac{\omega_{d}}{v_{d}}\left(1+z_{3}^{2}\right)\right), \frac{z_{4}}{k_{2}}\right]^{\top}$ and substituting (44), (45) and (43) into (48) yield

$$
\begin{aligned}
\dot{V}_{1}= & -k_{1}\left(z_{1}+z_{3}\left(z_{4}+\frac{\omega_{d}}{v_{d}}\left(1+z_{3}^{2}\right)\right)\right)^{2} \\
& -\frac{k_{3}}{k_{2}} z_{4}^{2}+f_{\eta}^{\top} T_{\alpha} \eta_{e} .
\end{aligned}
$$

If there is no $f_{\eta}^{\top} T_{\alpha} \eta_{e}$, then $\dot{V}_{1}$ is nonpositive. To eliminate this and ensure the desired system performance, we will design a dynamic controller in the next section.

3.2. Multiple dynamic controllers design. To cover all possible failure patterns, we will design one specific control law for each failure pattern. Then, we will also establish a control switching mechanism to select the appropriate control law to be applied.

3.2.1. Multiple dynamic control laws. Substituting (19) into (17), we have

$$
\dot{\eta}=-\bar{M}_{1}^{-1} \bar{M}_{2} \eta-\bar{M}_{1}^{-1} \bar{E}+\bar{M}_{1}^{-1} \bar{B} \sigma u,
$$

where $u=\left[u_{1 r}, u_{1 l}, u_{2 r}, u_{2 l}\right]^{\top}$ is the control signal applied. The time derivative of (42) is

$$
\dot{\eta}_{e}=-\bar{M}_{1}^{-1} \bar{M}_{2} \eta-\bar{M}_{1}^{-1} \bar{E}+\bar{M}_{1}^{-1} \bar{B} \sigma u-\dot{\eta}_{c} .
$$

Let $\sigma_{(k)}, k \in\{1,2, \ldots, N\}$ denote the $k$-th possible failure pattern matrix satisfying the condition (21), with $N$ being the number of all possible failure cases. Recalling (21), $\bar{M}_{1}^{-1} \bar{B} \sigma_{(k)}$ has full row rank, which also means its pseudo invertibility is guaranteed. For each $\sigma_{(k)}$, the control law is designed as

$$
\begin{aligned}
u_{(k)}= & \left(\bar{M}_{1}^{-1} \bar{B} \sigma_{(k)}\right)^{+}\left(-k_{4} \eta_{e}-T_{\alpha}^{\top} f_{\eta}\right. \\
& \left.+\bar{M}_{1}^{-1} \bar{M}_{2} \eta+\bar{M}_{1}^{-1} \bar{E}+\dot{\eta}_{c}\right),
\end{aligned}
$$

where $k_{4}>0$ is an arbitrarily chosen constant and $\left(\bar{M}_{1}^{-1} \bar{B} \sigma_{(k)}\right)^{+}$is a generalized inverse matrix satisfying $\bar{M}_{1}^{-1} \bar{B} \sigma_{(k)}\left(\bar{M}_{1}^{-1} \bar{B} \sigma_{(k)}\right)^{+}=I_{2}$. 
3.2.2. Performance analysis. Here the system performance is analyzed for each control law.

Lemma 1. If the control law applied matches the actual failure pattern matrix, i.e., $\sigma=\sigma_{(a)}$ and $u=u_{(a)}$ for $a \in\{1,2, \ldots, N\}$, then the boundedness of all closedloop signals is ensured, and $\lim _{t \rightarrow \infty}\left(x(t)-x_{d}(t)\right)=0$, $\lim _{t \rightarrow \infty}\left(y(t)-y_{d}(t)\right)=0, \lim _{t \rightarrow \infty}\left(\theta_{2}(t)-\theta_{d}(t)\right)=0$ and $\lim _{t \rightarrow \infty}\left(\eta(t)-\eta_{c}(t)\right)=0$, when $\left|\theta_{1}(0)-\theta_{2}(0)\right|<$ $\pi / 2$ and $\left|\theta_{2}(0)-\theta_{d}(0)\right|<\pi / 2$

Proof. Consider $\sigma=\sigma_{(a)}$ and $u=u_{(a)}$, and choose the Lyapunov function candidate as

$$
V_{2(a)}=V_{1}+\frac{1}{2} \eta_{e}^{\top} \eta_{e}
$$

Then, according to (49) and (51), its time derivative is

$$
\begin{aligned}
\dot{V}_{2(a)}= & -k_{1}\left(z_{1}+z_{3}\left(z_{4}+\frac{\omega_{d}}{v_{d}}\left(1+z_{3}^{2}\right)\right)\right)^{2}-\frac{k_{3}}{k_{2}} z_{4}^{2} \\
& +f_{\eta}^{\top} T_{\alpha} \eta_{e}+\eta_{e}^{\top}\left(-\bar{M}_{1}^{-1} \bar{M}_{2} \eta-\bar{M}_{1}^{-1} \bar{E}\right. \\
& \left.+\bar{M}_{1}^{-1} \bar{B} \sigma_{(a)} u-\dot{\eta}_{c}\right) \\
= & -k_{1}\left(z_{1}+z_{3}\left(z_{4}+\frac{\omega_{d}}{v_{d}}\left(1+z_{3}^{2}\right)\right)\right)^{2}-\frac{k_{3}}{k_{2}} z_{4}^{2} \\
& +\eta_{e}^{\top}\left(T_{\alpha}^{\top} f_{\eta}-\bar{M}_{1}^{-1} \bar{M}_{2} \eta-\bar{M}_{1}^{-1} \bar{E}\right. \\
& \left.+\bar{M}_{1}^{-1} \bar{B} \sigma_{(a)} u-\dot{\eta}_{c}\right) .
\end{aligned}
$$

Substituting (52) with $k=a$ into (54) yields

$$
\begin{aligned}
\dot{V}_{2(a)}= & -k_{1}\left(z_{1}+z_{3}\left(z_{4}+\frac{\omega_{d}}{v_{d}}\left(1+z_{3}^{2}\right)\right)\right)^{2} \\
& -\frac{k_{3}}{k_{2}} z_{4}^{2}-k_{4} \eta_{e}^{\top} \eta_{e} \leq 0
\end{aligned}
$$

which indicates $z_{1}, z_{2}, z_{3}, z_{4}, \eta_{e}, z_{1}+z_{3}\left(z_{4}+\frac{\omega_{d}}{v_{d}}(1+\right.$ $\left.\left.z_{3}^{2}\right)\right) \in L^{\infty}$, and $z_{4}, \eta_{e}, z_{1}+z_{3}\left(z_{4}+\frac{\omega_{d}}{v_{d}}\left(1+z_{3}^{2}\right)\right) \in L^{2}$.

From (33) and (34) it follows that $\cos e_{\theta} \neq 0$ and $\tan \left(\theta_{1}-\theta_{2}\right) \in L^{\infty}$, meaning $\cos \left(\theta_{1}-\theta_{2}\right) \neq 0$. Then, from Appendix C, (28)-(46) and (50)-(52), we can obtain that $T_{\alpha}$ is bounded and nonsingular, and $f_{\alpha}, \alpha_{c}, \alpha_{e}, \alpha, \eta_{c}, \eta, \dot{z}_{1}, \dot{z}_{2}, \dot{z}_{3}, \dot{z}_{4}, \dot{\alpha}_{c}, \dot{\eta}_{c}, u_{(k)}, \dot{\eta}, \dot{\eta}_{e}, \dot{\alpha}_{e}, \dot{\alpha}$ $\in L^{\infty}$, which also means that the time derivative of $z_{1}+z_{3}\left(z_{4}+\frac{\omega_{d}}{v_{d}}\left(1+z_{3}^{2}\right)\right)$ is bounded. According to Barbalat's lemma, it is concluded that all closed-loop signals are bounded, and $\lim _{t \rightarrow \infty}\left(z_{1}+z_{3}\left(z_{4}+\frac{\omega_{d}}{v_{d}}\left(1+z_{3}^{2}\right)\right)\right)=0, \lim _{t \rightarrow \infty} z_{4}=0$ and $\lim _{t \rightarrow \infty} \eta_{e}=0$, which also implies $\lim _{t \rightarrow \infty} \alpha_{e}=0$ and $\lim _{t \rightarrow \infty} \alpha_{c 1}=0$ with (43) and (44) meaning $\lim _{t \rightarrow \infty} \alpha_{1}=0$.

From (39), we have $\ddot{z}_{4}=\dot{\alpha}_{2}=\dot{\alpha}_{c 2}+\dot{\alpha}_{e 2} \in L^{\infty}$ with $\dot{\alpha}_{c 2}, \dot{\alpha}_{e 2} \in L^{\infty}$, which means that $\dot{z}_{4}$ is uniformly continuous, together with $\lim _{t \rightarrow \infty} \int_{0}^{t} \dot{z}_{4}(\tau) \mathrm{d} \tau=$ $z_{4}(\infty)-z_{4}(0)=-z_{4}(0)$. We can further obtain $\lim _{t \rightarrow \infty} \dot{z}_{4}=\lim _{t \rightarrow \infty} \alpha_{2}=\lim _{t \rightarrow \infty}\left(\alpha_{c 2}+\alpha_{e 2}\right)=0$ according to Barbalat's lemma. Then, with $\lim _{t \rightarrow \infty} \alpha_{e}=0, \lim _{t \rightarrow \infty} z_{4}=0, \alpha_{c 2}=-k_{2} v_{d} z_{3}-k_{3} z_{4}$ and $v_{d} \neq 0$, we have $\lim _{t \rightarrow \infty} \alpha_{c 2}=0$ and $\lim _{t \rightarrow \infty} z_{3}=0$. It follows that $\lim _{t \rightarrow \infty} z_{1}=0$ with $\lim _{t \rightarrow \infty}\left(z_{1}+z_{3}\left(z_{4}+\frac{\omega_{d}}{v_{d}}\left(1+z_{3}^{2}\right)\right)\right)=0$. On the other hand, from $\dot{z}_{3}=v_{d}\left(z_{4}-z_{2}\right)+\alpha_{1}\left(z_{4}-z_{2}+\frac{\omega_{d}}{v_{d}}\left(1+z_{3}^{2}\right)\right)$ in (38), we have $\ddot{z}_{3} \in L^{\infty}$. Similarly, $\lim _{t \rightarrow \infty} \dot{z}_{3}=0$ is ensured according to Barbalat's lemma. Then, we can further obtain $\lim _{t \rightarrow \infty} z_{2}=0$ with $\lim _{t \rightarrow \infty} z_{4}=0$, $\lim _{t \rightarrow \infty} \alpha_{1}=0$ and $v_{d} \neq 0$.

Finally, the boundedness of all closed-loop signals is ensured, $\lim _{t \rightarrow \infty} z_{i}(t)=0$, where $i=1,2,3,4$ and $\lim _{t \rightarrow \infty}\left(\eta(t)-\eta_{c}(t)\right)=0$, which also means $\lim _{t \rightarrow \infty}\left(x(t)-x_{d}(t)\right)=0, \lim _{t \rightarrow \infty}\left(y(t)-y_{d}(t)\right)=0$ and $\lim _{t \rightarrow \infty}\left(\theta_{2}(t)-\theta_{d}(t)\right)=0$ according to 31 - 33 and the transformation in (27).

Remark 4. For the controller parameters $k_{1}, \ldots, k_{4}$, choosing small ones may lead to a smooth system transient response but with a slow convergence speed of tracking errors, while choosing large ones may contribute to fast convergent tracking errors but with a large transient response. To fully utilize these properties, $k_{1}, \ldots, k_{4}$ are chosen empirically. The system may be first simulated with different sets of parameters (small ones and large ones); then we can choose the most appropriate set that may ensure a good smooth system transient response with an acceptable convergence speed of the tracking errors.

Since $\sigma$ is unknown, a control switching mechanism is needed to select the most appropriate $u_{(k)}$ from (52) as the employed control signal $u$.

3.3. Control switching mechanism design. In this subsection, we first reconstruct an $\eta_{(k)}$ for each possible $\sigma_{(k)}, k \in\{1,2, \ldots, N\}$. Then, multiple cost functions are calculated from the reconstruction errors and employed to generate the control switching signal.

3.3.1. Signal reconstruction. Consider the dynamic equation in (50):

$$
\dot{\eta}=-\bar{M}_{1}^{-1} \bar{M}_{2} \eta-\bar{M}_{1}^{-1} \bar{E}+\bar{M}_{1}^{-1} \bar{B} \sigma u .
$$

Choosing a stable filter $\frac{1}{s+\gamma}$ with $\gamma>0$ and operating both sides of (56), we have

$$
\begin{aligned}
\frac{s}{s+\gamma}[\eta](t)= & \frac{1}{s+\gamma}\left[-\bar{M}_{1}^{-1} \bar{M}_{2} \eta-\bar{M}_{1}^{-1} \bar{E}\right](t) \\
& +\frac{1}{s+\gamma}\left[\bar{M}_{1}^{-1} \bar{B} \sigma u\right](t),
\end{aligned}
$$

where $\dot{\eta}(t)=s[\eta](t)$ and $\frac{1}{s+\gamma}[\chi](t)$ denotes the output of the filter $\frac{1}{s+\gamma}$ with input $\chi(t)$ (Tao, 2003). From (57), we 
further obtain

$$
\begin{aligned}
\eta(t)= & \frac{\gamma}{s+\gamma}[\eta](t)+\frac{1}{s+\gamma}\left[-\bar{M}_{1}^{-1} \bar{M}_{2} \eta-\bar{M}_{1}^{-1} \bar{E}\right](t) \\
& +\frac{1}{s+\gamma}\left[\bar{M}_{1}^{-1} \bar{B} \sigma u\right](t)
\end{aligned}
$$

Now, for each possible $\sigma_{(k)}, k \in\{1,2, \ldots, N\}$, we reconstruct a signal as

$$
\begin{aligned}
\hat{\eta}_{(k)}(t)= & \frac{\gamma}{s+\gamma}[\eta](t)+\frac{1}{s+\gamma}\left[-\bar{M}_{1}^{-1} \bar{M}_{2} \eta-\bar{M}_{1}^{-1} \bar{E}\right](t) \\
& +\frac{1}{s+\gamma}\left[\bar{M}_{1}^{-1} \bar{B} \sigma_{(k)} u\right](t)
\end{aligned}
$$

Define the reconstruction error as

$$
\tilde{\eta}(t):=\eta(t)-\hat{\eta}(t) .
$$

Consider $\sigma=\sigma_{(a)}$. Then with (58) and (59), the matched reconstruction error is

$$
\tilde{\eta}_{(a)}(t)=0,
$$

and the unmatched reconstruction errors are

$$
\tilde{\eta}_{(b)}(t)=\frac{1}{s+\gamma}\left[\bar{M}_{1}^{-1} \bar{B} \sigma_{(a)} u-\bar{M}_{1}^{-1} \bar{B} \sigma_{(b)} u\right](t),
$$

for $b \in\{1,2, \ldots, N, b \neq a\}$, which may be nonzero.

3.3.2. Control signal selection. For control switching, multiple cost functions are first calculated from the reconstruction errors as

$$
J_{(k)}(t)=\tilde{\eta}_{(k)}^{\top}(t) \tilde{\eta}_{(k)}(t)
$$

for $k \in\{1,2, \ldots, N\}$. Then, control switching, as shown in Fig. 3, is implemented by comparing all the cost functions in (63), and determining the index $k$ corresponding to the minimum one, that is,

$$
k(t)=\arg \min _{k=1,2, \ldots, N} J_{(k)}(t),
$$

and then selecting the corresponding control law from (52) as the applied one, that is,

$$
u(t)=u_{(k)}(t) .
$$

3.4. Overall system performance analysis. This mobile robot system composed of two linked 2WD robots may be in different failure situations. To deal with these actuator failures, multiple control laws and multiple nonnegative cost functions are designed, each of which matches one possible failure situation. Control switching is implemented by comparing all the cost functions and applying the control law corresponding to the minimum one. The cost function matching the actual system is theoretically zero, which is minimum. Hence the matched control law will be selected, which can ensure system stability and asymptotic tracking properties. In some specific situations, an unmatched control law may be selected. This means that the corresponding unmatched cost function is minimum. In this case, the selected unmatched control law can also ensure the desired system performance. Therefore, the desired performance of the overall system is ensured by the developed multiple-model based control scheme.

The performance of the overall system is given as follows.

Theorem 1. The developed multiple-model actuator failure compensation control scheme, formed by the kinematic control law in (46), multiple dynamic control laws in (52) and the control switching mechanism implemented by (64) and (65) with multiple reconstructed signals in (59) and multiple cost functions in (63), applied to two linked 2 WD mobile robots modeled as (7) and (17), guarantees that all closed-loop signals are bounded and $\lim _{t \rightarrow \infty}\left(x(t)-x_{d}(t)\right)=0, \lim _{t \rightarrow \infty}\left(y(t)-y_{d}(t)\right)=0$, $\lim _{t \rightarrow \infty}\left(\theta_{2}(t)-\theta_{d}(t)\right)=0$, when $\left|\theta_{1}(0)-\theta_{2}(0)\right|<\pi / 2$ and $\left|\theta_{2}(0)-\theta_{d}(0)\right|<\pi / 2$, despite the presence of actuator failures modeled as (19) and (20).

Proof. Consider $\sigma=\sigma_{(a)}$. From 61) and 63), we have $J_{(a)}=0$ for the matched cost function. But for the unmatched functions $J_{(b)}, b \in\{1,2, \ldots, N, b \neq a\}$, this zero property may not hold due to 62. Since all cost functions are nonnegative, the matched cost function $J_{(a)}=0$ will generically become smaller than the other ones. Then, the matched control law $u_{(a)}$ will be selected as the employed one upon (64) and 65). According to Lemma 1 the selected control law can guarantee that all closed-loop signals are bounded and $\lim _{t \rightarrow \infty}(x(t)-$ $\left.x_{d}(t)\right)=0, \lim _{t \rightarrow \infty}\left(y(t)-y_{d}(t)\right)=0, \lim _{t \rightarrow \infty}\left(\theta_{2}(t)-\right.$ $\left.\theta_{d}(t)\right)=0$, despite the presence of actuator failures. This is the generic (generally true) matched case.

On the other hand, if the unmatched control law $u_{(b)}, b \neq a$, is selected as the employed one, which means $J_{(b)}(t) \leq J_{(a)}$, then $J_{(b)}(t)=0$ as $J_{(a)}(t)=$ 0 . From 62, $J_{(b)}(t)=0$ means $\bar{M}_{1}^{-1} \bar{B} \sigma_{(a)} u_{(b)}-$

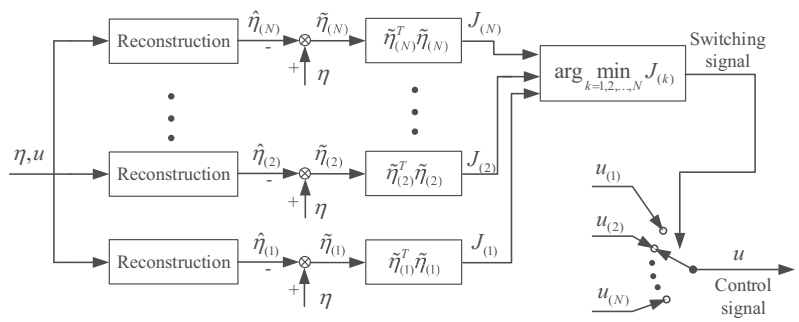

Fig. 3. Structure of the control switching mechanism. 
$\bar{M}_{1}^{-1} \bar{B} \sigma_{(b)} u_{(b)}=0$. According to (52), we further obtain $\bar{M}_{1}^{-1} \bar{B} \sigma_{(b)} u_{(b)}=\bar{M}_{1}^{-1} \bar{B} \sigma_{(a)} u_{(a)}$; it also means that if $\sigma=\sigma_{(a)}$ but $u_{(b)}$ is selected, then $u_{(b)}$ has the same control effectiveness compared with the matched control law $u_{(a)}$. In this sense, the desired system performance is also ensured for this unmatched case.

Remark 5. The designed multiple model control scheme can also be applied when some actuator failures occur and disappear, i.e., for intermittent failures. On the other hand, we would like to point out that the time intervals between every two different faulty cases should be long enough. This hypothesis means that the status of the actuators will not change quickly, which is also reasonable for the actual robots. Moreover, for practical robots, an artificial waiting time $T_{\min }>0$ (e.g., Narendra and Balakrishnan, 1997; Tan et al., 2016) may be employed between every two control switchings to prevent an arbitrarily fast switching. The proposed multiple-model control scheme only employs the switchings of control signals, but not a switched system that will be switched among several subsystems. On the other hand, the control switchings in this paper are independent of the waiting time, but they depend on the cost function based control switching mechanism. Thus we call $T_{\min }$ a waiting time but not a dwell-time that needs to be designed for switched systems.

Remark 6. In this paper, the control objective is focused on the tracking task for the rear robot, and the front robot can be seen to help the rear one to track the reference trajectory. The reason is the following: in this paper, the states $x, y$ are chosen to be the position of the rear robot, based on which the kinematics and dynamics are modeled, and then the control scheme is designed. Of course, the position of the front robot can also be chosen as the states $x$ and $y$, but the kinematic and dynamic models will change correspondingly. In this case, the control scheme should be redesigned.

\section{Simulation studies}

To verify the effectiveness of the developed multiple-model failure compensation scheme, a simulation study is presented as follows.

4.1. Simulation conditions. In this simulation, we assume that each of the two 2WD robots is the one used by Fukao et al. (2000); then the physical parameters are chosen as $a_{1}=a_{2}=0.3 \mathrm{~m}, b_{1}=b_{2}=0.75 \mathrm{~m}, r_{1}=$ $r_{2}=0.15 \mathrm{~m}, m_{1}=m_{2}=30 \mathrm{~kg}, I_{m 1}=I_{m 2}=15.625$ $\mathrm{kg} / \mathrm{m}^{2}$. The length of the link is assumed to be $d=1.7$ $\mathrm{m}$. In this simulation, an eight-like reference trajectory is considered. The velocities $v_{d}$ and $\omega_{d}$ are chosen as

$v_{d}=\sqrt{1.8225 \cos ^{2}(0.15 t)+1.265625 \cos ^{2}(0.075 t)} \frac{\mathrm{m}}{\mathrm{s}}$,

$\omega_{d}=\frac{f_{\omega_{d 1}}(t)-f_{\omega_{d 2}}(t)}{v_{d}^{2}} \frac{\mathrm{rad}}{\mathrm{s}}$,

where $f_{\omega_{d 1}}(t)=0.2278125 \sin (0.15 t) \cos (0.075 t)$, $f_{\omega_{d 2}}(t)=0.11390625 \sin (0.075 t) \cos (0.15 t)$. Then $x_{d}$, $y_{d}$ and $\theta_{d}$ are generated by (22)- (24) with $x_{d}(0)=$ $y_{d}(0)=\theta_{d}(0)=0$.

In order to verify failure compensation effectiveness of the developed multiple-model control scheme, the following failure cases are simulated:

no failure, $\sigma_{(1)}=\operatorname{diag}\{1,1,1,1\}, \quad 0 \leq t<100 \mathrm{~s}$, $\tau_{1 r}$ fails, $\quad \sigma_{(2)}=\operatorname{diag}\{0,1,1,1\}, 100 \mathrm{~s} \leq t<200 \mathrm{~s}$, $\tau_{1 r}, \tau_{2 r}$ fail, $\sigma_{(3)}=\operatorname{diag}\{0,1,0,1\}, 200 \mathrm{~s} \leq t<300 \mathrm{~s}$, $\tau_{2 r}, \tau_{2 l}$ fail, $\sigma_{(4)}=\operatorname{diag}\{0,1,1,0\}, 300 \mathrm{~s} \leq t<400 \mathrm{~s}$, $\tau_{1 r}, \tau_{2 l}$ fail, $\sigma_{(5)}=\operatorname{diag}\{1,1,0,0\}, t \geq 400 \mathrm{~s}$.

There are five failure pattern matrices that satisfy the condition (21): a failure free case, the one when one actuator fails, one actuator on the same side and different sides of each robot fails, and when the two actuators of Robot 2 fail. Then five control laws in (52), reconstructed signals in (59) and cost functions in (63) are employed. The initial conditions are chosen as $x(0)=0, y(0)=1$ $\mathrm{m}, \theta_{2}(0)=10 \mathrm{deg}, \theta_{1}(0)=0, v_{2}(0)=0$ and $\omega_{1}(0)=0$. The control gains are chosen as $k_{1}=1, k_{2}=2, k_{3}=0.5$ and $k_{4}=3$, and the waiting time between every two switchings is $T_{\min }=0.01 \mathrm{~s}$. Furthermore, considering a practical situation, in this simulation we also add some random sensor noise signals, whose variances are less than $(0.025)^{2}$.

4.2. Simulation results. The multiple-model failure compensation control scheme developed is applied. The following simulation results are given to demonstrate its effectiveness.

Figure 4 shows the trajectories of robot 2 (solid), the reference robot (dashed) and robot 1 (dot-dash), Fig. 5 shows the system tracking errors, and Fig. 6 shows the system velocities $v_{2}$ and $\omega_{1}$. Here, we can see that the desired system stability is ensured despite the presence of actuator failures and some random sensor noises, and even if the asymptotic tracking property is not ensured, the system tracking performance is good enough.

Figure 7 shows the control torques generated by the four wheels in two robots, from which we can see that the actuator failures are consistent with the failure cases in simulation conditions.

Figure 8 shows the control switching index, the dominating sequence of which is $1 \rightarrow 2 \rightarrow 3 \rightarrow 4 \rightarrow 5$. We can see that the control switching index matches with the actual failure pattern index. Although there are some 


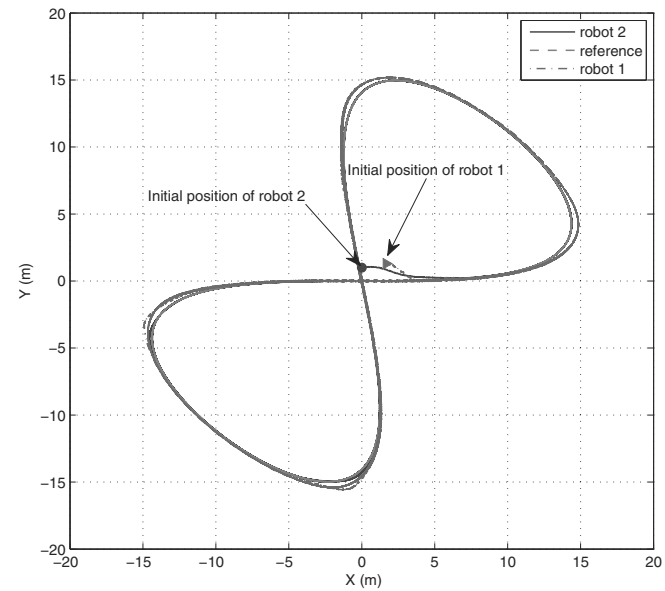

Fig. 4. Robot trajectories in the $(x, y)$ plane.
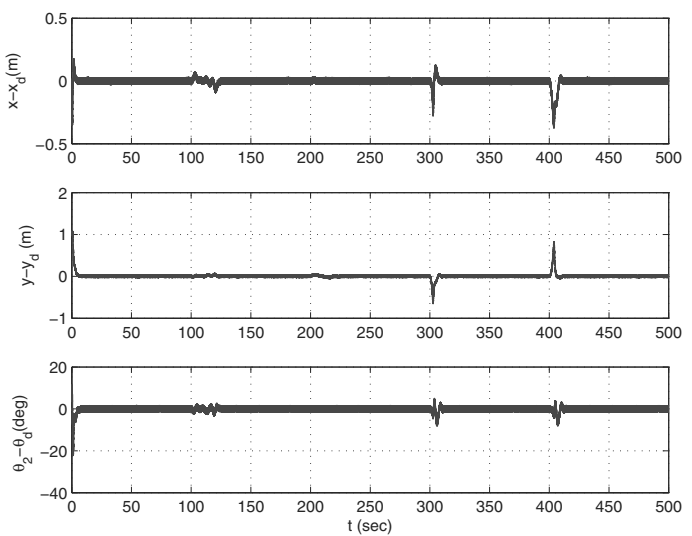

Fig. 5. Tracking errors: $x-x_{d}, y-y_{d}$ and $\theta_{2}-\theta_{d}$.
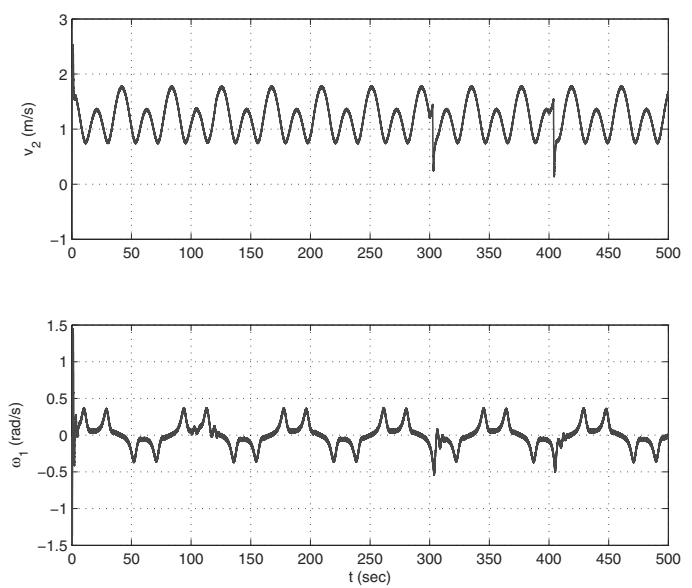

Fig. 6. Robot velocities: $v_{2}$ and $\omega_{1}$.
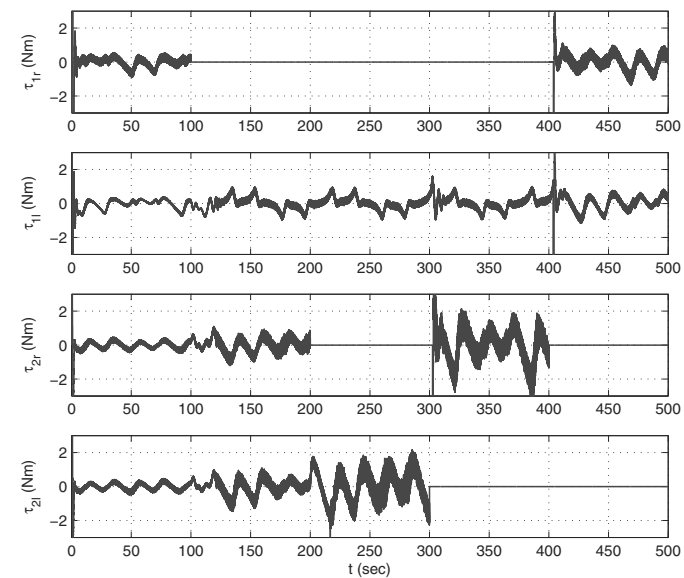

Fig. 7. Control torques generated by four actuated wheels.

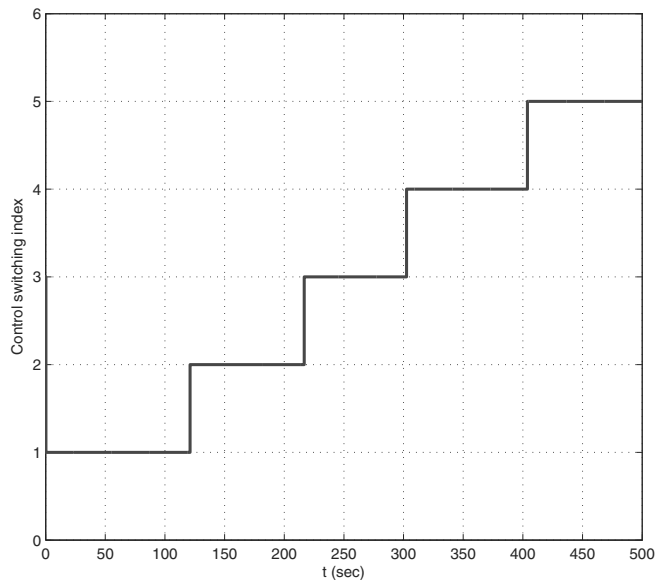

Fig. 8. Control switching index.

wrong or delayed switchings after failure occurrence time instants, which may be caused by the noisy measurements, the desired system performance is ensured. In addition, the control switching index can also be seen as additional failure identification information, because it generically matches the actual failure pattern index after a short time interval.

\section{Conclusions}

This paper developed a new actuator failure compensation system for two linked 2WD robots. The kinematics and dynamics of this two-robot configuration were modeled, thus a multiple-model failure compensation scheme was designed, including a kinematic control law, a multiple dynamic control law and a control switching mechanism. The effectiveness of the proposed failure compensation 
system was demonstrated by simulation results. Like all other multiple model control design techniques, although the simulation results show that the control switching settled down at one particular control signal in a finite time, this is not theoretically proven, which is still an open problem that needs to be solved. On the other hand, extending the proposed method for $n>2$ linked mobile robots is also our interest in future work.

\section{Acknowledgment}

This work was partially supported by the regional project SUCRé (Sûreté de fonctionnement et résilience pour la gestion et le contrôle coopératifs des systèmes sociotechniques: Coopération Homme(s)-Robot(s) en milieu hostile) of the Hauts-de-France region. The initial work for this paper was done when Yajie Ma was a post-doctoral fellow at the CRIStAL centre in France.

\section{References}

Akhtar, A., Nielsen, C. and Waslander, S. (2015). Path following using dynamic transverse feedback linearization for car like robots, IEEE Transactions on Robotics 31(2): 269-279.

Aref, M., Oftadeh, R., Ghabcheloo, R. and Mattila, J. (2015). Fault tolerant control architecture design for mobile manipulation in scientific facilities, International Journal of Advanced Robotic Systems 12(4): 1-12.

Bilski, A. and Wojciechowski, J. (2016). Automatic parametric fault detection in complex analog systems based on a method of minimum node selection, International Journal of Applied Mathematics and Computer Science 26(3): 655-668, DOI: 10.1515/amcs-2016-0045.

Blanke, M., Kinnaert, M., Lunze, J. and Staroswiecki, M. (2006). Diagnosis and Fault-Tolerant Control, Springer-Verlag, Berlin/Heidelberg.

Campion, G., Bastin, G. and D'Andrea-Novel, B. (1996). Structural properties and classification of kinematic and dynamic models of wheeled mobile robots, IEEE Transactions on Robotics and Automation 12(1): 47-62.

Canudas de Wit, C., NDoudi-Likoho, A. and Micaelli, A. (1997). Nonlinear control for a train-like vehicle, International Journal of Robotics and Research 16(3): 300-319.

Canudas de Wit, C., Siciliano, B. and Bastin, G. (2012). Theory of Robot Control, Springer-Verlag, London.

Caracciolo, L., Luca, A. and Iannitti, S. (1999). Trajectory tracking control of a four wheel differentially driven mobile robot, Proceedings of the 1999 IEEE International Conference on Robotics and Automation, Detroit, MI, USA, pp. 2632-2638.

Dixon, W., Dawson, D., Zergeroglu, E. and Behal, A. (2001). Adaptive tracking control of a wheeled mobile robot via an uncalibrated camera system, IEEE Transactions on Systems, Man, and Cybernetics B: Cybernetics 31(3): 341-352.
Do, K., Jiang, Z. and Pan, J. (2004). A global output-feedback controller for simultaneous tracking and stabilization of unicycle-type mobile robots, IEEE Transactions on Robotics and Automation 20(3): 589-594.

Efimov, D., Cieslak, J. and Henry, D. (2013). Supervisory fault-tolerant control with mutual performance optimization, International Journal of Adaptive Control and Signal Processing 17(4): 251-279.

Fierro, R. and Lewis, F. (1995). Control of a nonholonomic mobile robot: Backstepping kinematics into dynamics, Proceedings of the 34th Conference on Decision and Control, New Orleans, LO, USA, pp. 3805-3810.

Fourlas, G., Karras, G. and Kyriakopoulos, K. (2015). Fault tolerant control for a 4-wheel skid steering mobile robot, 26th International Workshop on Principles of Diagnosis, Paris, France, pp. 177-183.

Franzè, G., Tedesco, F. and Famularo, D. (2015). Actuator fault tolerant control: A receding horizon set-theoretic approach, IEEE Transactions on Automatic Control 80(8): 2225-2230.

Fukao, T., Nakagawa, H. and Adachi, N. (2000). Adaptive tracking control of a nonholonomic mobile robot, IEEE Transactions on Robotics and Automation 16(6): 609-615.

Ge, S., Wang, Z. and Lee, T. (2003). Adaptive stabilization of uncertain nonholonomic system by state and output feedback, Automatica 39(8): 1451-1460.

Goel, P., Dedeoglu, G., Roumeliotis, S. and Sukhatme, G. (2000). Fault detection and identification in a mobile robot using multiple estimation and neural network, Proceedings of the 2000 IEEE International Conference on Robotics and Automation, San Francisco, CA, USA, pp. 2302-2309.

González-Sierra, J., Aranda-Bricaire, E., Hernández-Mendoza, D. and Santiaguillo-Salinas, J. (2014). Emulation of $n$-trailer systems through differentially driven multi-agent systems: Continuous- and discrete-time approaches, Journal of Intelligent and Robotic Systems 75(1): 159-146.

Hamayun, M.T., Edwards, C., Alwi, H. and Bajodah, A. (2015). A fault tolerant direct control allocation scheme with integral sliding modes, International Journal of Applied Mathematics and Computer Science 25(1): 93-102, DOI: 10.1515/amcs-2015-0007.

Hassanabadi, A.H., Shafiee, M. and Puig, V. (2016). Robust fault detection of singular LPV systems with multiple time-varying delays, International Journal of Applied Mathematics and Computer Science 26(1): 45-61, DOI: 10.1515/amcs-2016-0004.

Huang, J., Wen, C., Wang, W. and Jiang, Z. (2014). Adaptive output feedback tracking control of a nonholonomic mobile robot, Automatica 50(3): 821-831.

Ji, M. and Sarkar, N. (2007). Supervisory fault adaptive control of a mobile robot and its application in sensor-fault accommodation, IEEE Transactions on Robotics 23(1): 174-178.

Ji, M., Zhang, Z., Biswas, G. and Sarkar, N. (2003). Hybrid fault adaptive control of a wheeled mobile robot, IEEE/ASME Transactions on Mechatronics 8(2): 226-233. 
Khalaji, A. and Moosavian, S. (2014). Robust adaptive controller for a tractor-trailer mobile robot, IEEE/ASME Transactions on Mechatronics 19(3): 943-953.

Kim, T., Park, J. and Kim, H. (2015). Actuator reconfiguration control of a robotic vehicle with four independent wheel driving, 15th International Conference on Control, Automation and Systems, Busan, Korea, pp. 1767-1770.

Koh, M., Noton, M. and Khoo, S. (2012). Robust fault-tolerant leader-follower control of four-wheel-steering mobile robots using terminal sliding mode, Australian Journal of Electrical and Electronics Engineering 9(4): 247-254.

Kozłowski, K. and Pazderski, D. (2004). Modeling and control of a 4-wheel skid-steering mobile robot, International Journal of Applied Mathematics and Computer Science 14(4): 477-496.

Li, X. and Yang, G. (2012). Robust adaptive fault-tolerant control for uncertain linear systems with actuator failures, IET Control Theory and Applications 6(10): 1544-1551.

Michałek, M. (2014). A highly scalable path-following controller for N-trailers with off-axle hitching, Control Engineering Practice 29: 61-73.

Michałek, M. (2017). Cascade-like modular tracking controller for non-standard N-trailer, IEEE Transactions on Control System Technology 25(2): 619-627.

Morin, P. and Samson, C. (2012). Feedback control of the general two-trailers system with the transverse function approach, IEEE 51st Annual Conference on Decision and Control, Maui, HI, USA, pp. 1003-1010.

Narendra, K. and Balakrishnan, J. (1997). Adaptive control using multiple-models, IEEE Transactions on Automatic Control 42(2): 171-187.

Patton, R., Chen, L. and Klinkhieo, S. (2012). An LPV pole-placement approach to friction compensation as an FTC problem, International Journal of Applied Mathematics and Computer Science 22(1): 149-160, DOI: 10.2478/v10006-012-0011-z.

Ritzen, P., Roebroek, E., Van de Wouw, N., Jiang, Z. and Nijmeijer, H. (2016). Trailer steering control of a tractor-trailer robot, IEEE Transactions on Control System Technology 24(4): 1240-1252.

Rotondo, D., Nejjari, F. and Puig, V. (2015). Robust quasi-LPV model reference FTC of a quadrotor UAV subject to actuator faults, International Journal of Applied Mathematics and Computer 25(1): 7-22, DOI: 10.1515/amcs-2015-0001.

Rotondo, D., Puig, V., Nejjari, F. and Romera, J. (2014). A fault-hiding approach for the switching quasi-LPV fault-tolerant control of a four-wheeled omnidirectional mobile robot, IEEE Transactions on Industrial Electronics 62(6): 3932-3944.

Skoundrianos, E. and Tzafestas, S. (2004). Finding fault: Fault diagnosis on the wheels of a mobile robot using local model neural networks, IEEE Robotics and Automation Magazine 11(3): 83-90.
Sørdalen, O. and Wichlund, K. (1993). Exponential stabilization of a car with $n$ trailers, 32 nd Conference on Decison Control, San Antonio, TX, USA, pp. 978-983.

Tan, C., Yang, H. and Tao, G. (2016). A multiple-model MRAC scheme for multivariable systems with matching uncertainties, Information Sciences 360(10): 217-230.

Tao, G. (2003). Adaptive Control Design and Analysis, John Wiley \& Sons, Hoboken, NJ.

Tilbury, D., Sørdalen, O., Bushnell, L. and Sastry, S. (1995). A multisteering trailer system: Conversion into chained form using feedback, IEEE Transactions on Robotics and Automation 11(6): 807-818.

Yang, H., Fan, X., Shi, P. and Hua, C. (2016). Nonlinear control for tracking and obstacle avoidance of a wheeled mobile robot with nonholonomic constraint, IEEE Transactions on Control Systems Technology 24(2): 741-746.

Yang, X. and Maciejowski, J. (2015). Fault tolerant control using Gaussian processes and model predictive control, International Journal of Applied Mathematics and Computer Science 25(1): 133-148, DOI: 10.1515/amcs-2015-0010.

Ye, D. and Yang, G. (2006). Adaptive fault-tolerant tracking control against actuator faults with application to flight control, IEEE Transactions on Control Systems Technology 14(6): 1088-1096.

Yu, X. and Jiang, J. (2015). A survey of fault-tolerant controllers based on safety-related issues, Annual Reviews in Control 39: 46-57.

Yuan, J., Sun, F. and Huang, Y. (2015). Trajectory generation and tracking control for double-steering tractor-trailer mobile robots with on-axle hitching, IEEE/ASME Transactions on Mechatronics 62(12): 7665-7677.

Zhang, X. and Cocquempot, V. (2014). Fault tolerant control for an electric 4WD vehicle's path tracking with active fault diagnosis, 19th IFAC World Congress, Cape Town, South Africa, pp. 6728-6734.

Zhang, Y. and Jiang, J. (2008). Bibliographical review on reconfigurable fault-tolerant control systems, Annual Reviews in Control 32(2): 229-252.

Zou, A. and Kumar, K. (2012). Robust attitude coordination control for spacecraft formation flying under actuator failures, AIAA Journal of Guidance, Control and Dynamics 35(4): 1247-1255.

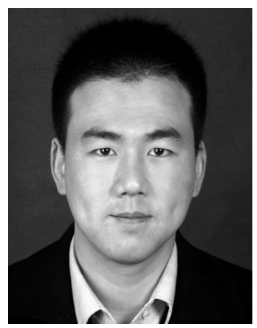

Yajie Ma received the BSc degree in automation from the Nanjing University of Aeronautics and Astronautics, China, in 2008, the MS degree in detection technology and automatic equipment from Hohai University, Nanjing, in 2011, and the $\mathrm{PhD}$ degree in control theory and control engineering from the Nanjing University of Aeronautics and Astronautics in 2015. From 2015 to 2016, he was a post-doctoral fellow of the Research Center in Computer Science, Signal Processing and Automatic Control (CRIStAL-CNRS UMR 9189), Lille, France. Currently, he is a lecturer at the College of Automation Engineering with the Nanjing University of Aeronautics and Astronautics. His research interests include adaptive fault diagnosis and fault tolerant control, and their applications. 


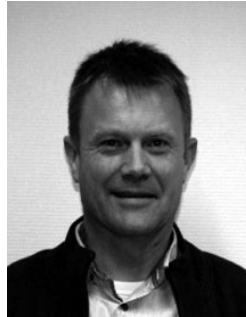

Vincent Cocquempot received the $\mathrm{PhD}$ degree in automatic control from the Lille 1 University of Sciences and Technologies, France, in 1993. $\mathrm{He}$ is currently a professor of automatic control and computer science at the Technological Institute of the Lille University of Sciences and Technologies, and a researcher at CRIStAL-CNRS UMR 9189, Lille, France. His research interests include robust on-line fault diagnosis for uncertain dynamical nonlinear systems, fault detection and isolation, and fault tolerant control for hybrid dynamical systems.

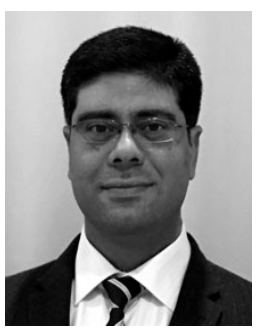

Maan El Badaoui El Najjar received the BSc degree in computer engineering from ESISARINP Grenoble, France, in 1999, the MSc degree in control system from ENSIEG-INP Grenoble in 2000, and the PhD degree in perception and control systems from the University of Technology of Compiegne in 2003. He is currently a professor at the University of Lille, and the head of the team on diagnostics, control and observation for fault tolerant systems (DiCOT) in the CRIStAL laboratory. His research interests include fault tolerant multi-sensor fusion to deal with faulty sensors, and its application to robotics and intelligent vehicle systems.

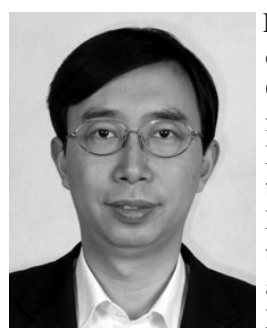

Bin Jiang received the $\mathrm{PhD}$ degree in automatic control from Northeastern University, Shenyang, China, in 1995 . He is currently a chair professor with the Cheung Kong Scholar Program, Ministry of Education, China, and the dean of the College of Automation Engineering with the Nanjing University of Aeronautics and Astronautics. His research interests include fault diagnosis and fault-tolerant control and their applications. Prof. Jiang currently serves as an associate editor or an editorial board member of IEEE Transactions on Control Systems Technology, the International Journal of Control, Automation and Systems, and others. He is the chair of the Control Systems Chapter of the IEEE Nanjing Section.

\section{Appendix A}

\section{Derivation of (28)-(30)}

With (25)-(27), we have

$$
\begin{aligned}
& e_{x}=\left(x-x_{d}\right) \cos \theta_{d}+\left(y-y_{d}\right) \sin \theta_{d}, \\
& e_{y}=-\left(x-x_{d}\right) \sin \theta_{d}+\left(y-y_{d}\right) \cos \theta_{d}, \\
& e_{\theta}=\theta_{2}-\theta_{d} .
\end{aligned}
$$

Together with (3)-(5) and (22)-(24), we have

$$
\begin{aligned}
\dot{e}_{x}= & \left(v_{2} \cos \theta_{2}-v_{d} \cos \theta_{d}\right) \cos \theta_{d}-\left(x-x_{d}\right) \omega_{d} \sin \theta_{d} \\
& +\left(v_{2} \sin \theta_{2}-v_{d} \sin \theta_{d}\right) \sin \theta_{d}+\left(y-y_{d}\right) \omega_{d} \cos \theta_{d} \\
= & \omega_{d}\left(-\left(x-x_{d}\right) \sin \theta_{d}+\left(y-y_{d}\right) \cos \theta_{d}\right) \\
& +v_{2}\left(\cos \theta_{2} \cos \theta_{d}+\sin \theta_{2} \sin \theta_{d}\right)
\end{aligned}
$$

$$
\begin{aligned}
& -v_{d}\left(\cos \theta_{d} \cos \theta_{d}+\sin \theta_{d} \sin \theta_{d}\right) \\
= & \omega_{d} e_{y}+v_{2} \cos e_{\theta}-v_{d}, \\
\dot{e}_{y}= & -\left(v_{2} \cos \theta_{2}-v_{d} \cos \theta_{d}\right) \sin \theta_{d}-\left(x-x_{d}\right) \omega_{d} \cos \theta_{d} \\
& +\left(v_{2} \sin \theta_{2}-v_{d} \sin \theta_{d}\right) \cos \theta_{d}-\left(y-y_{d}\right) \omega_{d} \sin \theta_{d} \\
= & \omega_{d}\left(-\left(x-x_{d}\right) \cos \theta_{d}-\left(y-y_{d}\right) \sin \theta_{d}\right) \\
& +v_{2}\left(-\cos \theta_{2} \sin \theta_{d}+\sin \theta_{2} \cos \theta_{d}\right) \\
& +v_{d}\left(\sin \theta_{d} \cos \theta_{d}-\sin \theta_{d} \sin \theta_{d}\right) \\
= & -\omega_{d} e_{x}+v_{2} \sin e_{\theta}, \\
\dot{e}_{\theta}= & \frac{v_{2}}{d} \tan \left(\theta_{1}-\theta_{2}\right)-\omega_{d} .
\end{aligned}
$$

\section{Appendix B}

\section{Derivation of (36)-(39)}

It is easy to obtain $\dot{z}_{1}, \dot{z}_{2}$ and $\dot{z}_{4}$ from (28)-35). From $z_{3}=\tan e_{\theta}$ in (33) and with (32), (34) and (35), we have

$$
\begin{aligned}
\dot{z}_{3}= & \frac{1}{\cos ^{2} e_{\theta}} \dot{e}_{\theta}=\frac{v_{2} \tan \left(\theta_{1}-\theta_{2}\right)}{d \cos ^{2} e_{\theta}}-\frac{\omega_{d}}{\cos ^{2} e_{\theta}} \\
= & \frac{\tan \left(\theta_{1}-\theta_{2}\right)}{d \cos ^{3} e_{\theta}} v_{2} \cos e_{\theta}-\frac{\omega_{d}}{v_{d} \cos ^{2} e_{\theta}} v_{2} \cos e_{\theta} \\
& +\frac{\omega_{d}}{v_{d} \cos ^{2} e_{\theta}}\left(v_{2} \cos e_{\theta}-v_{d}\right) \\
= & \left(z_{4}-z_{2}\right) v_{2} \cos e_{\theta}+\frac{\omega_{d}}{v_{d} \cos ^{2} e_{\theta}} \alpha_{1} \\
= & v_{d}\left(z_{4}-z_{2}\right)+\alpha_{1}\left(z_{4}-z_{2}+\frac{\omega_{d}}{v_{d}}\left(1+z_{3}^{2}\right)\right) .
\end{aligned}
$$

\section{Appendix C}

\section{Derivation of $T_{\alpha}$ and $f_{\alpha}$ in (40)}

From

$$
z_{4}=\frac{\tan \left(\theta_{1}-\theta_{2}\right)}{d \cos ^{3} e_{\theta}}-\frac{\omega_{d}}{v_{d} \cos ^{2} e_{\theta}}+e_{y}
$$

in (34), and with (5), (6), 30) and (29), we have

$$
\begin{aligned}
\dot{z}_{4}= & \frac{\frac{\dot{\theta}_{1}-\dot{\theta}_{2}}{\cos ^{2}\left(\theta_{1}-\theta_{2}\right)} \cos ^{3} e_{\theta}+3 \tan \left(\theta_{1}-\theta_{2}\right) \cos ^{2} e_{\theta} \sin e_{\theta} \dot{e}_{\theta}}{d \cos ^{6} e_{\theta}} \\
- & \frac{\dot{\omega}_{d} v_{d} \cos ^{2} e_{\theta}-\omega_{d}\left(\dot{v}_{d} \cos ^{2} e_{\theta}-2 v_{d} \cos e_{\theta} \sin e_{\theta} \dot{e}_{\theta}\right)}{v_{d}^{2} \cos ^{4} e_{\theta}} \\
& +\dot{e}_{y} \\
= & \frac{\omega_{1}-\frac{v_{2}}{d} \tan \left(\theta_{1}-\theta_{2}\right)}{d \cos ^{3} e_{\theta} \cos ^{2}\left(\theta_{1}-\theta_{2}\right)}-\frac{\dot{\omega}_{d}}{v_{d} \cos ^{2} e_{\theta}}+\frac{\omega_{d} \dot{v}_{d}}{v_{d}^{2} \cos ^{2} e_{\theta}} \\
& +\frac{3 \tan \left(\theta_{1}-\theta_{2}\right) \sin e_{\theta}\left(\frac{v_{2}}{d} \tan \left(\theta_{1}-\theta_{2}\right)-\omega_{d}\right)}{d \cos ^{4} e_{\theta}} \\
& -\frac{2 \omega_{d} \sin e_{\theta}\left(\frac{v_{2}}{d} \tan \left(\theta_{1}-\theta_{2}\right)-\omega_{d}\right)}{v_{d} \cos ^{3} e_{\theta}} \\
& -\omega_{d} e_{x}+v_{2} \sin e_{\theta}
\end{aligned}
$$




$$
\begin{aligned}
= & \left(\frac{3 \tan ^{2}\left(\theta_{1}-\theta_{2}\right) \sin e_{\theta}}{d^{2} \cos ^{4} e_{\theta}}-\frac{\tan \left(\theta_{1}-\theta_{2}\right)}{d^{2} \cos ^{3} e_{\theta} \cos ^{2}\left(\theta_{1}-\theta_{2}\right)}\right. \\
& \left.-\frac{2 \omega_{d} \tan \left(\theta_{1}-\theta_{2}\right) \sin e_{\theta}}{d v_{d} \cos ^{3} e_{\theta}}+\sin e_{\theta}\right) v_{2} \\
& +\frac{1}{d \cos ^{3} e_{\theta} \cos ^{2}\left(\theta_{1}-\theta_{2}\right)} \omega_{1}-\frac{\dot{\omega}_{d}}{v_{d} \cos ^{2} e_{\theta}} \\
& -\frac{3 \omega_{d} \tan \left(\theta_{1}-\theta_{2}\right) \sin e_{\theta}}{d \cos ^{4} e_{\theta}} \\
& +\frac{\omega_{d} \dot{v}_{d}}{v_{d}^{2} \cos ^{2} e_{\theta}}+\frac{2 \omega_{d}^{2} \sin e_{\theta}}{v_{d} \cos ^{3} e_{\theta}}-\omega_{d} e_{x},
\end{aligned}
$$

Together with $\alpha_{1}=v_{2} \cos e_{\theta}-v_{d}$ and $\alpha_{2}=\dot{z}_{4}$. we can finally obtain

$$
\begin{aligned}
\alpha & =\left[\begin{array}{l}
\alpha_{1} \\
\alpha_{2}
\end{array}\right]=T_{\alpha} \eta+f_{\alpha} \\
& =\left[\begin{array}{ll}
T_{\alpha 11} & T_{\alpha 12} \\
T_{\alpha 21} & T_{\alpha 22}
\end{array}\right]\left[\begin{array}{l}
v_{2} \\
\omega_{1}
\end{array}\right]+\left[\begin{array}{l}
f_{\alpha 1} \\
f_{\alpha 2}
\end{array}\right],
\end{aligned}
$$

where $T_{\alpha 11}=\cos e_{\theta}, T_{\alpha 12}=0$,

$$
\begin{aligned}
T_{\alpha 21}= & \frac{3 \tan ^{2}\left(\theta_{1}-\theta_{2}\right) \sin e_{\theta}}{d^{2} \cos ^{4} e_{\theta}}-\frac{\tan \left(\theta_{1}-\theta_{2}\right)}{d^{2} \cos ^{3} e_{\theta} \cos ^{2}\left(\theta_{1}-\theta_{2}\right)} \\
& -\frac{2 \omega_{d} \tan \left(\theta_{1}-\theta_{2}\right) \sin e_{\theta}}{d v_{d} \cos ^{3} e_{\theta}}+\sin e_{\theta}, \\
T_{\alpha 22}= & \frac{1}{d \cos ^{3} e_{\theta} \cos ^{2}\left(\theta_{1}-\theta_{2}\right)}, f_{\alpha 1}=-v_{d}, \\
f_{\alpha 2}= & -\frac{3 \omega_{d} \tan \left(\theta_{1}-\theta_{2}\right) \sin e_{\theta}}{d \cos ^{4} e_{\theta}}-\frac{\dot{\omega}_{d}}{v_{d} \cos ^{2} e_{\theta}} \\
& +\frac{\omega_{d} \dot{v}_{d}}{v_{d}^{2} \cos ^{2} e_{\theta}}+\frac{2 \omega_{d}^{2} \sin e_{\theta}}{v_{d} \cos ^{3} e_{\theta}}-\omega_{d} e_{x} .
\end{aligned}
$$

Received: 25 September 2016 Revised: 29 April 2017

Re-revised: 25 June 2017 Accepted: 4 July 2017 